\title{
New engineering treatment of bovine pericardium confers outstanding resistance to calcification in mitral and pulmonary implantations in a juvenile sheep model
}

\author{
Christian P. Brizard, MD, MS,,${ }^{a, b, c}$ Johann Brink, MBBS, ${ }^{a}$ Steven B. Horton, PhD,,${ }^{a, b, c}$ \\ Glenn Anthony Edwards, BVSc, MANZCSc, ${ }^{\mathrm{d}} \mathrm{John}$ C. Galati, PhD, BSc, ${ }^{\mathrm{b}, \mathrm{e}}$ and \\ William M. L. Neethling, PhD, FACA ${ }^{\mathrm{f}, \mathrm{g}}$
}

\begin{abstract}
Objective: To conduct a test of noninferiority for CardioCel (Admedus, Brisbane, Australia), a chemically engineered bovine pericardium over autologous pericardium treated intraoperatively with glutaraldehyde in a chronic juvenile sheep model of pulmonary valve (PV) and mitral valve (MV) reconstruction.
\end{abstract}

\begin{abstract}
Methods: We replaced the posterior leaflet of the MV and of 1 PV cusp with patches in ewes aged 10 months. There were 2 groups: CardioCel $(n=6)$ and control $(n=4)$. All valves were competent. Echocardiography was performed before euthanasia. The collected data were function, macroscopy, histology, and calcium contents. The primary end points were thickening and calcium content.
\end{abstract}

\begin{abstract}
Results: All animals survived until sacrifice after 7 months. The valves had normal echo. The macroscopic aspect of the valves was excellent. Examination of the slides for both groups revealed a continuous endothelium on both sides of the patch and a layer of new collagen developed on both sides between patch and endothelium and interstitial cells and smooth muscle cell in these layers. The patch had not thickened but the 2 layers of new collagen for the PV showed a median thickening of $37 \%$ in the CardioCel group and $111 \%$ in the control group $(P=.01)$, and for the MV a thickening of $108 \%$ and $251 \%$, respectively, was seen $(P=.01)$. The median calcium content in the PV was $0.24 \mu \mathrm{g} / \mathrm{mg}$ (range, 0.19-0.30) in the CardioCel group versus $0.34 \mu \mathrm{g} / \mathrm{mg}$ (range, 0.24-0.62) in controls $(P=.20)$. In the MV it was $0.46 \mu \mathrm{g} / \mathrm{mg}$ (range, 0.30-1.0) in the CardioCel group and $0.47 \mu \mathrm{g} / \mathrm{mg}$ (range, $0.29-1.9)$ in controls $(P=1.0)$.
\end{abstract}

Conclusions: In this growing lamb model the CardioCel patch allowed accurate valve repair at both systemic and pulmonary pressure. The mechanical properties of CardioCel after 7 months were preserved with a more controlled healing than the treated autologous pericardium and without calcification. (J Thorac Cardiovasc Surg 2014;148:3194-201)

See related commentary on pages 3202-3.

From the Cardiac Surgery Department, ${ }^{\text {a }}$ Royal Children's Hospital, Melbourne, Victoria, Australia; Murdoch Childrens Research Institute, ${ }^{\mathrm{b}}$ Parkville, Victoria, Australia; Department of Pediatrics ${ }^{\mathrm{c}}$ and School of Veterinary Science, ${ }^{\mathrm{d}}$ University of Melbourne, Parkville, Victoria, Australia; Department of Mathematics and Statistics, ${ }^{\mathrm{e}}$ La Trobe University, Melbourne, Victoria, Australia; and Fremantle Heart Institute ${ }^{\mathrm{f}}$ and Department of Cardiothoracic Surgery, ${ }^{\mathrm{g}}$ Fremantle Hospital, School of Surgery, University of Western Australia, Perth, Western Australia, Australia.

Supported by the Heart Research Group of the Royal Children's Hospital, Melbourne, with the assistance of a grant from Admedus, Brisbane, Australia.

Disclosures: Christian P. Brizard reports consulting and lecture fees from Admedus. Johann Brink reports consulting fees from Admedus. William M. L. Neethling reports consulting fees from Admedus. Glenn Anthony Edwards reports lecture fees from the Center for Veterinary Medicine at the Food and Drug Administration. All other authors have nothing to disclose with regard to commercial support.

Read at The American Association for Thoracic Surgery Mitral Conclave, New York, New York, May 2-3, 2013.

Received for publication June 3, 2014; revisions received July 31, 2014; accepted for publication Aug 1, 2014; available ahead of print Sept 11, 2014

Address for reprints: Christian P. Brizard, MD, MS, Cardiac Surgery Department, Royal Children's Hospital, Parkville 3052, Victoria, Australia (E-mail: christian. brizard@ @rch.org.au).

$0022-5223 / \$ 36.00$

Copyright $(\odot) 2014$ by The American Association for Thoracic Surgery

http://dx.doi.org/10.1016/j.jtcvs.2014.08.002
Our department is heavily involved in pediatric valve repair. ${ }^{1-3}$ Despite a long history of trial and error and extensive clinical and experimental research in our unit and others, ${ }^{4}$ the ideal material for valve tissue replacement and extension is yet to be found. As in many units, for more than 2 decades our best default material for valve repair has been autologous pericardium treated intraoperatively with $0.625 \%$ glutaraldehyde. ${ }^{5}$

In 2009 we were introduced to CardioCel (Admedus, Brisbane, Australia), a pericardium patch developed and chemically engineered in Australia. The multistep ADAPT treatment ([Admedus] decellularized with Tx-100; deoxycholate, IgePal CA-630; and denucleased, crosslinked in $0.05 \%$ monomeric glutaraldehyde and detoxified) applied to bovine pericardium confers to the treated patch complete fixation, ${ }^{6}$ fine handling properties, and outstanding resistance to calcification. ${ }^{6,7}$

Before committing to use of this novel product for valve repair in pediatric patients, we reviewed in vivo studies performed in subcutaneous juvenile rat models first against human pericardium rapidly cross-linked with $0.6 \%$ glutaraldehyde for 4 minutes and detoxified with magnesium 
chloride, and with human pericardium exposed to the multistep ADAPT treatment. ${ }^{8}$ Both studies showed the CardioCel product to have complete cross-linking and extreme resistance to calcification. These characteristics were far superior to the ones of the current default materials used in the unit.

The purpose of our study was to compare in a juvenile sheep model the autologous pericardial patch cross-linked intraoperatively versus CardioCel in a clinical situation. Both the pulmonary valve and the mitral valve were partially replaced with the materials studied. The model has been used in the past for the mitral valve position (Michel Pellerin and Alain Carpentier, personal communication, 2011) and discriminates for the mechanical properties and calcification of the patch. In our model we added the partial replacement of the pulmonary valve to evaluate the mechanical properties of CardioCel in the semilunar location.

\section{METHODS}

This was a prospective, nonrandomized study performed as a test of noninferiority of the CardioCel patch to the autologous pericardium cross-linked in the operating theater with glutaraldehyde $0.625 \%$. Primary end points were thickening and calcium content. Secondary end points were echographic evaluation, macroscopic evaluation, histology, and immunohistochemistry. The 4 animals of the control group received autologous pericardium patches treated intraoperatively with glutaraldehyde. The 6 animals of the study group received CardioCel patches. The order of the surgeries was as follows: the first 2 animals received CardioCel, then 1 control, 4 additional animals received CardioCel, and the 3 final animals were control over a 6-week period.

All animals received humane care in compliance with the Principles of Laboratory Care (National Society for Medical Research) and the Guide for the Care and Use of Laboratory Animals. The Animal Ethics Committee of the University of Melbourne approved the study protocol. The surgery was performed at the Surgery and Biomaterial Research Unit in the University of Melbourne Veterinary Hospital, Werribee, and the animals were kept in the same location afterward.

\section{Surgical Preparation}

The animals were anesthetized and prepped according to a previously published protocol. ${ }^{9}$ The sheep were premedicated with buprenorphine $(0.01 \mathrm{mg} / \mathrm{kg})$ before induction of anesthesia with intravenous propofol $(4 \mathrm{mg} / \mathrm{kg}$ ). An endotracheal tube was placed and the cuff inflated. Anesthesia was maintained with a mixture of isoflurane (1.5\%-2\%) and oxygen via an anesthetic machine. Fentanyl citrate $(0.4 \mu \mathrm{g} / \mathrm{kg} / \mathrm{min})$ was used while the sheep were on cardiopulmonary bypass. Paralysis was induced with intravenous atracurium $0.5 \mathrm{mg} / \mathrm{kg}$ initially followed by boluses of $0.1-0.2 \mathrm{mg} / \mathrm{kg}$ every hour or as required. The sheep were mechanically ventilated at $1.5 \mathrm{~L} / \mathrm{min}, 25 \mathrm{~cm}$ water pressure $8 \mathrm{bpm}, 80$ to $100 \mathrm{~mL}$ tidal volume. A wide bore stomach tube was placed orally to evacuate the fluid of the rumen during the procedure to prevent aspiration of ruminal content. Amiodarone $5 \mathrm{mg} / \mathrm{kg}$ was administered to prevent arrhythmias.

A left thoracotomy in the third intercostal space was performed with division of the fourth costal cartilage. Cardiopulmonary bypass at $34^{\circ}$ was instituted between the descending aorta and the body of the right atrium cannulated through the right atrial appendage. In the control group, a $4 \times 4$ $\mathrm{cm}$ pericardial patch was harvested; trimmed of fat; treated with $0.625 \%$ glutaraldehyde for 8 minutes; and rinsed in 3 baths of normal saline, the last 1 containing magnesium. ${ }^{4}$ In the CardioCel group, the pericardium was available from the container and used without rinsing or washing.
Under crossclamp and using repeated warm blood cardioplegia, the left atrium was incised parallel to the atrioventricular groove and the mitral valve was exposed. The posterior leaflet was incised $1 \mathrm{~mm}$ inside the annulus and as much as possible of the body of the posterior leaflet was removed without compromising the marginal chordae. An ellipsoid patch was tailored and inserted using running 6-0 polypropylene suture material. The valve was tested with saline inflation. The left atrium was closed. Then the antero-left aspect of the main pulmonary artery was incised longitudinally going deep into the corresponding sinus. Under direct vision, the antero-left cusp was carefully excised following the hinge line. The cusp was used as a template for tailoring the pericardial replacement and including the free edge. The pericardial cusp was secured with running 5-0 polypropylene suture material. The pulmonary valve was tested with saline. The pulmonary artery was closed and the heart deaired. Bypass was terminated when the temperature reached $38^{\circ} \mathrm{C}$. The chest was closed on 1 chest drain that was removed several hours after the lamb was back in its pen. Heparin was given for 24 hours.

\section{Follow-up Protocol}

The animals were kept in their pen onsite for the duration of the study. Five months are required to adhere to the recommendations of ISO 10993-1:2009-Biological evaluation of medical device. Our aim was to close and complete the study once calcifications were visible on echocardiography in either group or after 12 months. At 8 months after the initial procedure, 2 control animals were anesthetized and the left thoracotomy was reopened. Epicardial echocardiography was performed and enhanced echogenicity of the mitral patch was seen in both animals (Figure 1, A). The decision was made to put to death all animals with 2 CardioCel animals also having a general anesthesia induction and an epicardial echocardiographic study, whereas the other animals were put to death with a lethal injection of phenobarbital.

\section{Macroscopic Examination, Histology, and Calcium Analyses}

The hearts were explanted and the valves were dissected out en bloc. The macroscopic aspect and pliability of the valves and the patches were evaluated. The valves were labelled and photographed. The valves were then fixed in $10 \%$ buffered formalin and stored in that solution. The tissues were included in paraffin and $0.5-\mu \mathrm{m}$ sections prepared for histology stains that included hematoxylin and eosin, trichrome, Von Kossa, and picrosirius red. Immunohistochemistry was performed in both groups on some histology sections and included Factor VIII, smooth muscle actin, and vimentin. The pathologist who reviewed the slides was blinded to the groups. The thickening above and below the patch was calculated after measuring the total area of thickening over a specific length and expressed in micrometers (micrometers squared thickening divided by micrometer length of valve tissue). The thickening was reported to the thickness of the patch as measured on the slide and expressed in percent of the later. Calcium content was measured by an independent assessor by means of inductively coupled plasma mass spectrometry using a Varian Vista atomic emission spectrometer with axial viewing and a continuing calibration blank (Varian Australia Pty Ltd, Victoria, Australia).

Precise limited and identical area or the valves were used for histology section, whereas the remaining tissue was used for calcium content measurement. Calcium content was also measured in samples of surrounding native valve tissue of the host animal.

\section{Statistical Analysis}

Prism software version 5.0 (exact graphs and data analysis) statistical package for windows (GraphPad Software, La Jolla, Calif) and Stata 12 (Statcorp, College Station, Tex) were used for statistical analysis. Quantitative data were presented as median and range. Nonparametric $\chi^{2}$ test of medians was used to compare CardioCel and control groups, with results cross-checked against the more conservative Fisher exact test. Median 


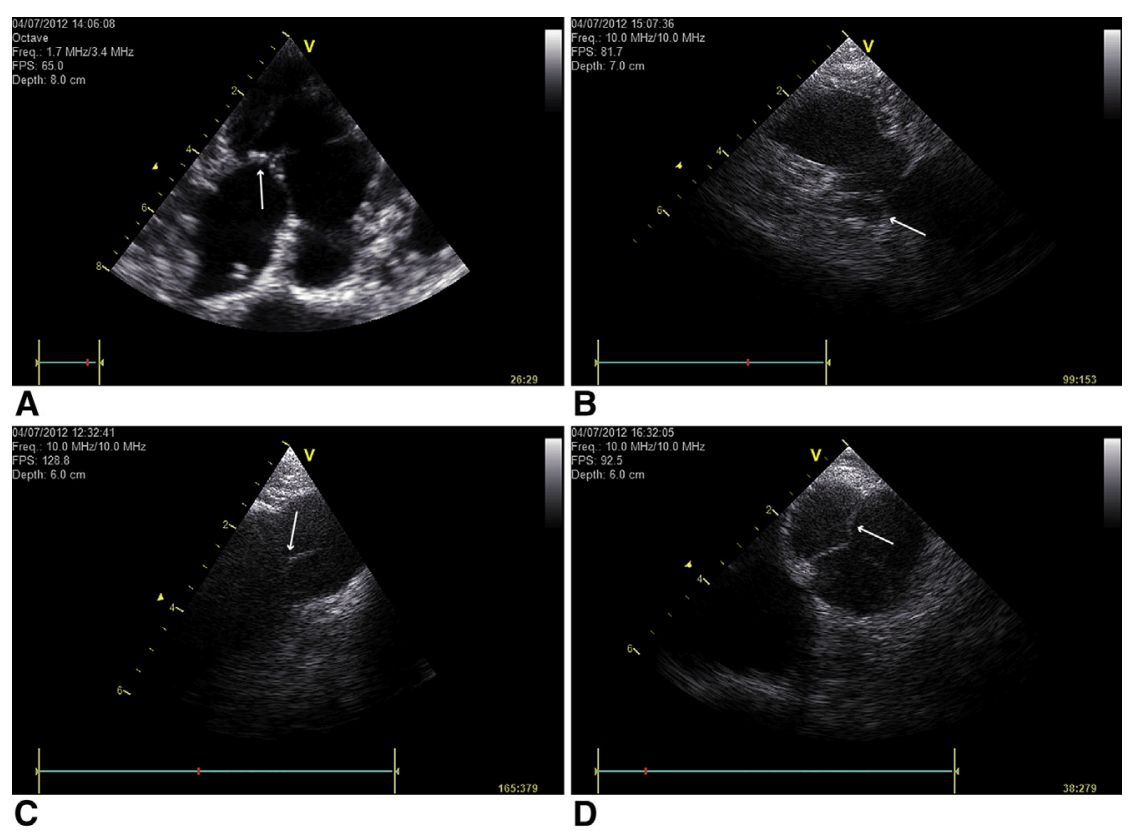

FIGURE 1. Epicardial echocardiography. A, Long axis study of the mitral valve. The white arrow points at the posterior leaflet bearing the hyperechogenic autologous pericardial patch. B, Long axis study of the mitral valve. The white arrow points at the posterior leaflet that does not display hyperechogenicity. $\mathrm{C}$, Long axis of the pulmonary valve. The white arrow points at the unremarkable autologous patch. $\mathrm{D}$, Short axis of the pulmonary valve. The white arrow points at the CardioCel patch, thin and flexible.

calcium content in valves compared with surrounding tissue was compared using a nonparametric matched-pairs sign test.

\section{RESULTS}

Ten Corriedale Merino ewes (aged 10 months), median weight $32.5 \mathrm{~kg}$ (range, 27.5-40 kg), were operated on. All animals survived without complications.

\section{Epicardial Echocardiographic Studies}

Two control animals (out of 4) and 2 study animals (out of 6) underwent epicardial echocardiography after dissection of the adherences under general anesthesia. In the 2 control animals, the echocardiographic study demonstrated enhanced echogenicity with shadowing in both mitral valve patches and on 1 pulmonary valve patch. One pulmonary valve had trivial regurgitation. In the study group, both animals were free of calcification on both sites. In both groups, the echocardiographic aspect was of thin and pliable patches (Figure 1, A-D).

\section{Macroscopic Examination}

In all animals and in both locations, the surface of the patches was smooth and without evidence of macroscopic thrombi attached or visible calcification. The aspect was very satisfactory. There was no sign of retraction or dilation and no perforation was detectable (Figure 2, A-D).

\section{Slides}

In all groups and in both sites, 3 layers could be distinctly identified using conventional stain and immunohistochemistry.
In all patches, 2 outer layers made of organized collagen and containing very few cells were covering each side of the pericardial patch, representing the middle layer. Hematoxylin and eosin slides are shown in Figure 3, A-D. In both groups, a continuous endothelial lining was visible on the external blood interface and confirmed with Factor VIII presence (Figure 4, A-D).

Picrosirius red stains collagen I (fibers) in deep yellow and red birefringence, whereas collagen III (fibrils), which are less mature and less organized, display green birefringence. ${ }^{10}$

The slides of the control group mitral valves showed a high density of collagen I in the native patch in the middle layer, whereas the outer layers were twice as thick and very loose with a low density of collagen I. Low intensity green staining is visible in either layer (Figure 5, $A$ ).

The mitral valves in the CardioCel group patches show a high density of collagen I in the patch and in the outer layers. However, the outer layers are thin and regular, half the thickness of the patch itself. Collagen III is visible in the patch and to a lesser degree in the outer layers (Figure 5, B).

The pulmonary valves slides show a similar pattern with a very thin layer of predominantly collagen I laid on each side of the patch for the CardioCel group, whereas the control group patches were deeply imbedded between thick layers of collagen I and III. The overall thickness of the control group patches, including the superior and inferior layers, were twice the thickness of the CardioCel patch (Figure 5, $C$ and $D$ ). 

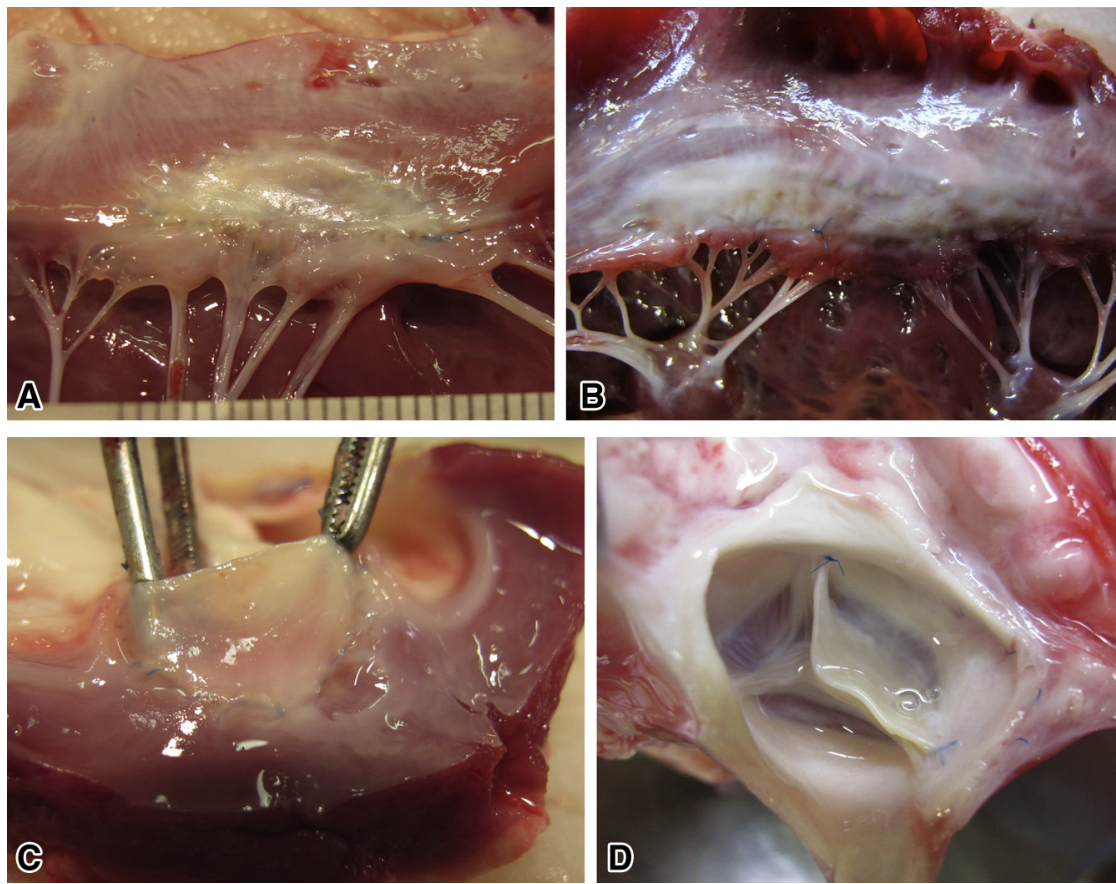

FIGURE 2. Macroscopy. A and B, both the autologous and the CardioCel patches have the same macroscopic aspect. C and D, Both the autologous and the CardioCel are flexible. The CardioCel patch after 8 months implantation is remarkably comparable to a fresh patch.

In all groups, few neocapillaries, valve interstitial cells (myofibroblasts), some monocytes, and cells with smooth muscle cells phenotype (confirmed with smooth muscle $\alpha$ actin stain; photo not shown) were visible. Trichrome stain is shown in Figure 6, $A-D$.
In the control group only, rare calcifications were seen using Von Kossa stain (photo not shown). However, only part of the patches in both group were available for section and staining, the remaining having been kept for the calcium measurements.
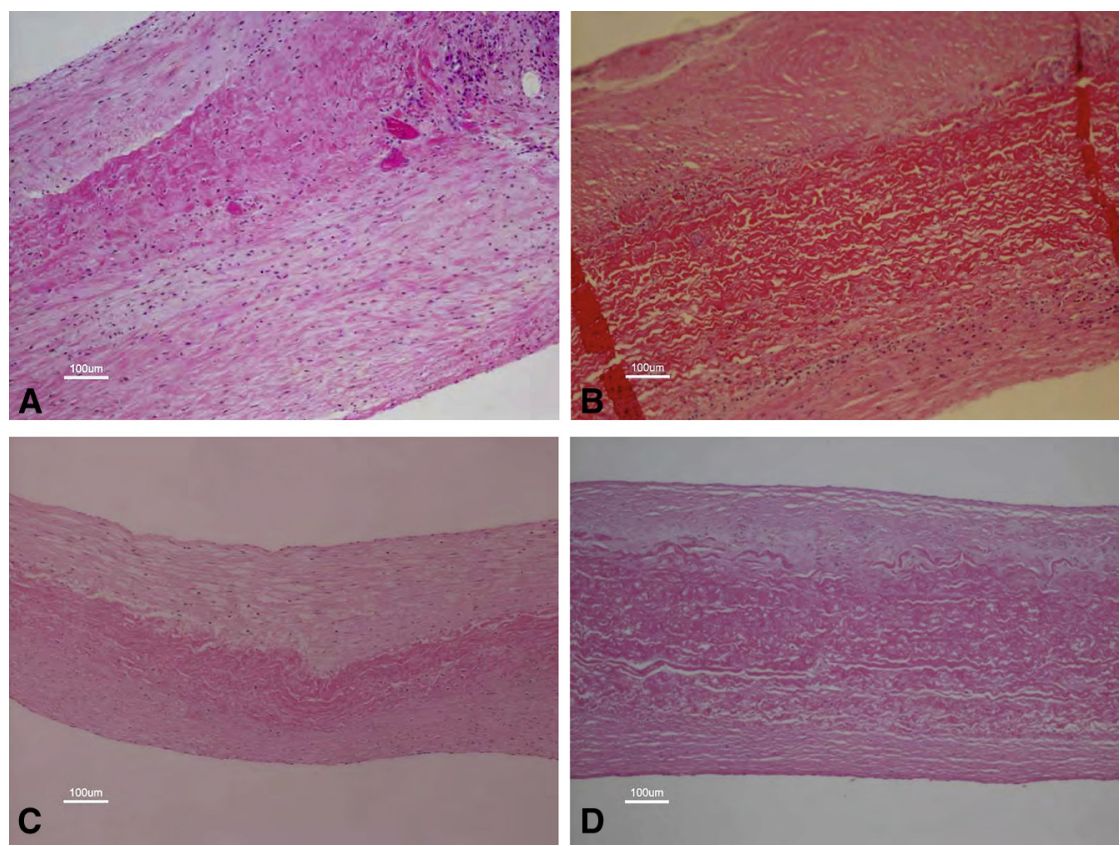

FIGURE 3. Hematoxylin and eosin. A, B, C, and D, All slides show a central layer made of the original patch and 2 outer layers of new collagen. An endothelial layer is continuous on the outer surface of the new collagen layers. Some mononucleated cells and lymphocytes can be identified in the autologous group mostly the mitral slide (A). 

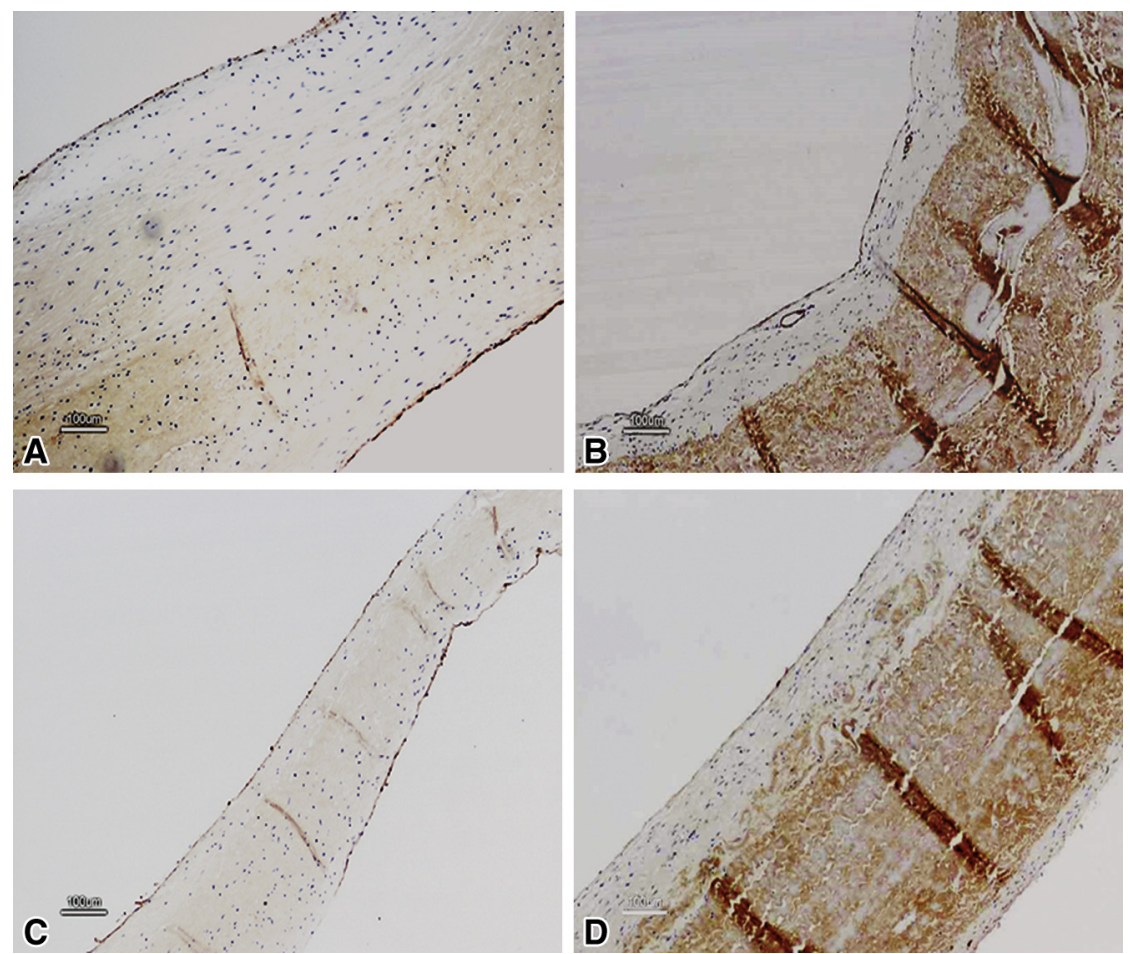

FIGURE 4. Factor VIII immunohistochemistry. A, B, C, and D, The presence of a continuous endothelium in all groups on the outer margin of the neocollagen layers.

\section{Thickness}

The thickness of the explanted patches was compared with the thickness at the time of implantation. The CardioCel patches were $0.4 \mathrm{~mm}$ thick at the time of implantation (manufacturer's specification). The thickness was identical on the explanted CardioCel patches measured on the slides (median $0.398 \mathrm{~mm}$ and $0.389 \mathrm{~mm}$ ) in the mitral and pulmonary valve positions, respectively (Table 1).
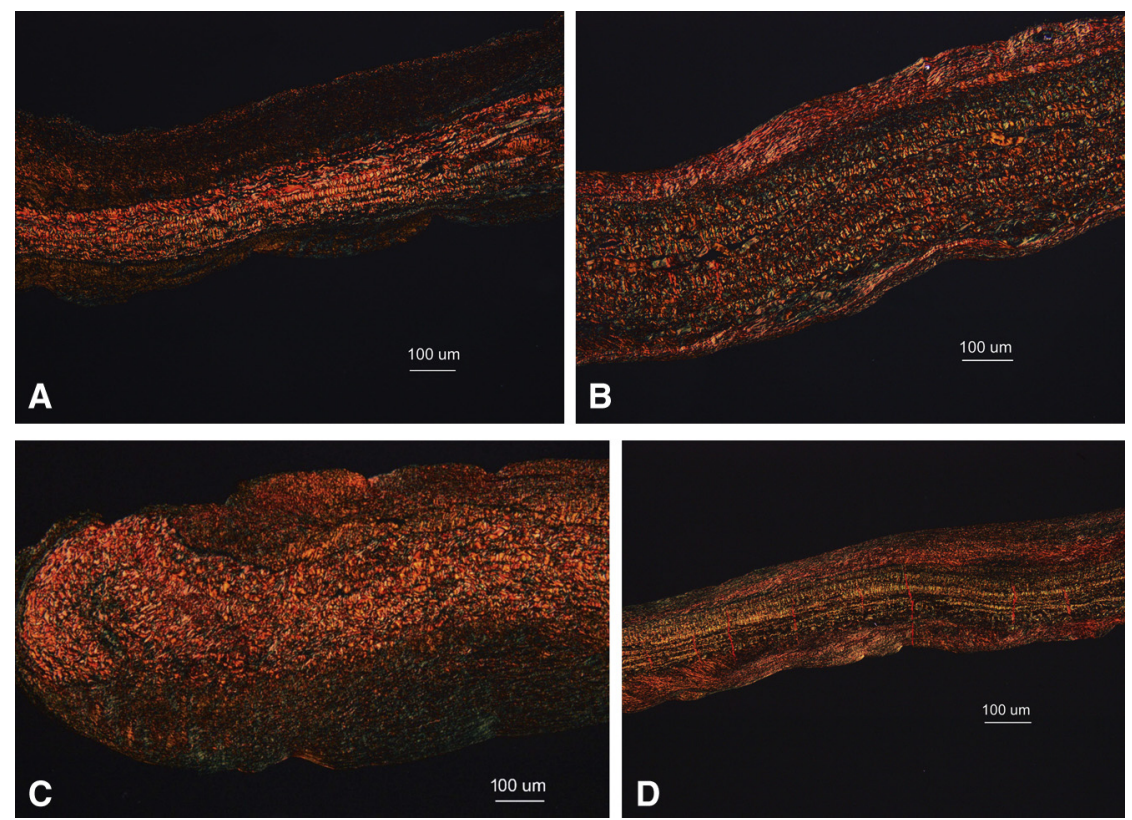

FIGURE 5. Picrosirius red. In the control group ([A] mitral and [C] pulmonary) the outer layers are made of new collagen, predominantly collagen I, of the same thickness than the central layer (autologous pericardial patch). The outer layers are much less dense than the autologous patch and contain some immature collagen III in green. In the CardioCel (Admedus, Brisbane, Australia) group ([B] mitral and [D] pulmonary, the outer layers are very dense but thin and made exclusively of collagen I. 

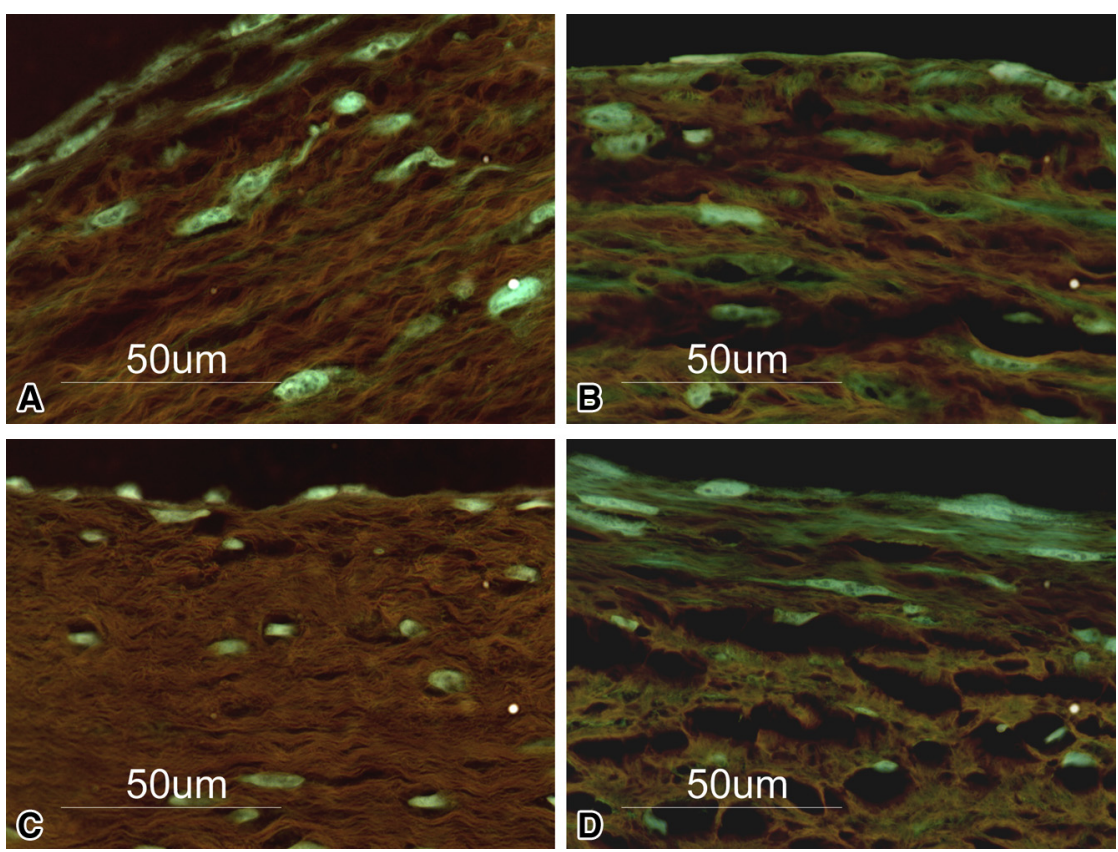

FIGURE 6. Trichrome staining with differential color adjustment demonstrating endothelial cells on the surface followed by a mixture of smooth muscle cells (confirmed by the smooth muscle cell $\alpha$ actin immunohistochemistry; photo not shown) and interstitial cells in the newly formed collagen. A and C, Control groups. B and D, CardioCel (Admedus, Brisbane, Australia). Note the difference in the distribution of the cells between groups. (High contrast light microscopy with; original magnification $\times 100$ ).

The autologous pericardial patch thickness at the time of explantation was measured on the slides-median 0.206 $\mathrm{mm}$ and $0.159 \mathrm{~mm}$ for mitral and pulmonary patches, respectively - but the thickness at the time of implantation could not be measured. As found in the CardioCel group, it was assumed that the patch itself in the control group had not thickened.

\section{Thickening}

The thickness of the superior and inferior layers of new collagen (the thickening) was measured. The combined thickness of the superior and inferior layers of new collagen was reported to the thickness of the patch at the time of explantation for both positions and both groups (Table 1) and expressed in percent of the latter. For the pulmonary valves the median thickening was $37 \%$ in the CardioCel group and $111 \%$ in the control group $(P=.01)$. For the mitral valves the median thickening was $108 \%$ in the CardioCel group and $251 \%$ in the control group $(P=.01)$.

\section{Extractable Calcium Contents}

The median calcium content in the pulmonary valves was $0.34 \mu \mathrm{g} / \mathrm{mg}$ (range, $0.24-0.62 \mu \mathrm{g} / \mathrm{mg}$ ) of dry tissue in the control group versus $0.24 \mu \mathrm{g} / \mathrm{mg}$ (range, $0.19-0.30$ $\mu \mathrm{g} / \mathrm{mg})$ in the CardioCel group $(P=.20)$ (Tables 2 and 3). In the mitral valves the median calcium content was 0.47 $\mu \mathrm{g} / \mathrm{mg}$ (range, $0.29-1.9 \mu \mathrm{g} / \mathrm{mg}$ ) in the control group and $0.46 \mu \mathrm{g} / \mathrm{mg}$ (range, $0.30-1.0 \mu \mathrm{g} / \mathrm{mg}$ ) in the CardioCel group $(P=1.0)$. The median calcium content of the valvular tissue surrounding the repair was higher in all groups compared with the calcium content of the patches themselves. The difference was more pronounced in the CardioCel pulmonary valves (median, 0.87 vs $0.24 ; P=.03$ ).

\section{DISCUSSION \\ Interpretation of the Data}

The purpose of our study was to evaluate the behavior of the patch over the long-term; the testing of the cross-linking having been conducted and published before. ${ }^{6}$ Tensile

TABLE 1. Thickness and thickening

\begin{tabular}{|c|c|c|c|c|}
\hline $\operatorname{Group}(\mathbf{N}=20)$ & Mitral control $(n=4)$ & Mitral CardioCel $(n=6)$ & Pulmonary control $(n=6)$ & Pulmonary CardioCel $(n=6)$ \\
\hline Initial patch thickness (mm) & NA & 0.4 & NA & $0.4^{*}$ \\
\hline Patch thickness on the slide (mm) & $0.206(0.17-0.3)$ & $0.398(0.3-0.49)$ & $0.159(0.12-0.31)$ & $0.389(0.31-0.45)$ \\
\hline Thickness of new collagen (mm) & $0.55(0.36-0.63)$ & $0.43(0.2-0.56)$ & $0.24(0.12-0.66)$ & $0.15(0.12-0.24)$ \\
\hline Thickening ratio $(\%)$ & $251(162-286)$ & $108(50-139)$ & $111(53-297)$ & $38(31-59)$ \\
\hline$P$ & \multicolumn{2}{|r|}{$.01 *$} & \multicolumn{2}{|r|}{$.01 \dagger$} \\
\hline
\end{tabular}

Values are given as median (range) unless otherwise noted. NA, Not applicable. *Fisher exact test $P=.048$ for mitral control versus mitral CardioCel (Admedus, Brisbane, Australia). $\dagger$ Fisher exact test $P=.048$ for pulmonary control versus pulmonary CardioCel. 
TABLE 2. Calcium content in the mitral valve patches and surrounding valve tissue

\begin{tabular}{|c|c|c|c|c|}
\hline Group & Mitral control $(n=4)$ & Mitral CardioCel $(n=6)$ & Mitral surrounding control $(n=4)$ & $\begin{array}{c}\text { Mitral surrounding } \\
\text { CardioCel }(n=6)\end{array}$ \\
\hline Calcium ( $\mu \mathrm{g} / \mathrm{mg})$ & $0.47(0.29-1.87)$ & $0.46(0.3-1.0)$ & $0.63(0.58-0.71)$ & $0.62(0.35-0.77)$ \\
\hline$P$ & & & & \\
\hline
\end{tabular}

Values are given as median (range). *Mitral CardioCel (Admedus, Brisbane, Australia) versus control.

properties were determined by an independent assessor (study report No. 061210R1, Helm-Holtz Institute, Aachen, Germany). Acute resistance to systemic pressure (ultimate strength) of the CardioCel patch has been compared with the ultimate strength of the human aorta and aortic valve cusp. The ultimate strength in 11 CardioCel samples was $9.18 \pm 0.54 \mathrm{~N} / \mathrm{mm}^{2}$, exceeding the longitudinal ultimate tensile strength of the normal human ascending aorta $\left(1.8 \pm 0.24 \mathrm{~N} / \mathrm{mm}^{2}\right)^{11}$ and the circumferential ultimate tensile strength of the normal human aortic cusp $\left(1.71 \pm 0.14 \mathrm{~N} / \mathrm{mm}^{2}\right)^{12}$ by a magnitude of more than 5-fold.

In our study, both patches behave very satisfactorily at the 8 months horizon. All mitral valves were competent and 1 pulmonary valve had trivial incompetence in the control group. Both patches demonstrated healing, lining each side with a layer of new collagen covered with continuous endothelial cells. The composition of the layer of new collagen varied between the control group and the CardioCel group. The collagen I was thin and dense in the CardioCel group. In the control group the collagen I was thick and less dense in the pulmonary position and even less so in the mitral position. Hardly any collagen III was visible in the CardioCel group, whereas some was visible in the control group both in the patch and in the outer layers. The picrosirius red slides suggest that in the control group a low-grade remodeling process with production of collagen III was occurring in the native patch while the healing process in the outer layers was still active. In the CardioCel group, the picrosirius red suggests that the healing process was controlled and completed with thin and organized dense collagen I and hardly any collagen III.

There were very low calcium concentrations in both groups, confirming the resistance to calcification of the autologous pericardium treated extemporarily. However, there was superior resistance to calcification in the CardioCel patch, particularly in the pulmonary position. The calcium level in the native tissue valve surrounding the patch was consistently higher than in the patch in both groups, with the difference more pronounced in the CardioCel study group. This finding confirms the resistance to calcification, and also signifies that the patches host essentially too few cells to demonstrate evidence of calcium metabolism.

The minimal calcification level and the absence of lymphocytes together with the quality of the healing process suggest a mild and insignificant immune response by the host to the patch. ${ }^{13}$ Both are less with the bovine pericardium treated with ADAPT then with the conventional intraoperative treatment of the autologous pericardium, confirming in this model the findings of the subcutaneous implantation in rats. ${ }^{6,8}$

\section{COMMENTS}

The aim of our study was first to evaluate the CardioCel patch in the valvular setting at systemic and pulmonary pressure and second to compare it with the default state-of-theart material for pediatric valve repairs. We deliberately used for control autologous pericardia treated intraoperatively with glutaraldehyde instead of other commercially available regular bovine pericardia. As demonstrated with our own subcutaneous model in rats and by others, ${ }^{8}$ a standard glutaraldehyde-treated bovine pericardium patch performs much less satisfactorily than the intraoperatively treated autologous pericardium. This has also been demonstrated in human clinical practice in our center and by others. ${ }^{14}$ In our practice as in many other units, use of standard commercially available treated bovine pericardia was abandoned many years ago.

We do not use untreated autologous pericardium apart from the pulmonary sinus reconstruction in arterial switch. ${ }^{15}$ Despite unfavorable experimental data suggesting retraction and thickening, ${ }^{16}$ some units use it routinely for aortic valve repair. ${ }^{17}$

Therefore, we used for control the material currently most apt for valve repair in pediatric patients and indeed the 1 less likely to be inferior to the CardioCel patch.

The end points of our study were clinical (ie, function of the valve, histology, inflammation, and evidence of host response) and chemical (ie, calcification level).

Despite the complexity of the model, perfect initial outcome was achieved with both materials. All animals

TABLE 3. Calcium content in the pulmonary valve patches and surrounding valve tissue

\begin{tabular}{lcccc}
\hline \multicolumn{1}{c}{ Group } & Pulmonary control $(\mathbf{n}=\mathbf{4})$ & Pulmonary CardioCel $(\mathbf{n}=\mathbf{6})$ & $\begin{array}{c}\text { Pulmonary surrounding } \\
\text { control }(\mathbf{n}=\mathbf{4})\end{array}$ & $\begin{array}{c}\text { Pulmonary surrounding } \\
\text { CardioCel }(\mathbf{n}=\mathbf{6})\end{array}$ \\
\hline $\begin{array}{l}\text { Calcium }(\mu \mathrm{g} / \mathrm{mg}) \\
P^{*}\end{array}$ & $0.34(0.24-0.62)$ & $0.24(0.19-0.3)$ & $0.9(0.3-2.53)$ & $0.87(0.62-0.92)$ \\
\hline Values are given as median (range). ${ }^{*} \chi^{2}$ rank sum test of median. $\dagger$ Pulmonary CardioCel (Admedus, Brisbane, Australia) versus control. $\ddagger$ CardioCel versus surrounding.
\end{tabular}


survived to the term of the study. Both materials performed to the expected outcome result with competent valves and normal echocardiographic function of the patch in the animals tested. This demonstrates the noninferiority of the CardioCel patch for the clinical end points during the time span studied. As for the microscopic- and calciumlevel primary end points, the CardioCel patch has significantly improved results for thickening and less calcification in the pulmonary position. Calcification is equivalent in the mitral position with a smaller standard deviation. In both sites, the calcium level is significantly less than in the surrounding tissue. The histologic studies demonstrate that CardioCel generates a more controlled and gentle healing process despite a greater collagen mass (as suggested by the initial patch thickness) than in the autologous pericardium in this infant lamb model.

\section{CONCLUSIONS}

In this rare, long-term animal model, CardioCel compared very favorably to state-of-the-art autologous pericardium treated intraoperatively with glutaraldehyde in terms of valve function, both in the systemic and pulmonary pressure regimen. A controlled, low-grade, and harmonious healing process was demonstrated. The patch is associated with extremely low calcium deposition, lower than in the control autologous pericardium in the pulmonary position. These findings suggest that the very long-term mechanical properties of the patch should be preserved in clinical situations.

\section{References}

1. Oppido G, Davies B, McMullan DM, Cochrane AD, Cheung MMH, d'Udekem Y, et al. Surgical treatment of congenital mitral valve disease: midterm results of a repair-oriented policy. J Thorac Cardiovasc Surg. 2008; 135:1313-20; discussion 1320-1.

2. Siddiqui J, Brizard CP, Galati JC, Iyengar AJ, Hutchinson D, Konstantinov IE, et al. Surgical valvotomy and repair for neonatal and infant congenital aortic stenosis achieves better results than interventional catheterization. J Am Coll Cardiol. 2013;62:2134-40.
3. McMullan DM, Oppido G, Davies B, Kawahira Y, Cochrane AD, d'Udekem d'Acoz Y, et al. Surgical strategy for the bicuspid aortic valve: tricuspidization with cusp extension versus pulmonary autograft. J Thorac Cardiovasc Surg. 2007; 134:90-8.

4. Vincentelli A, Zegdi R, Prat A, Lajos P, Latremouille C, LeBret E, et al. Mechanical modifications to human pericardium after a brief immersion in 0.625\% glutaraldehyde. J Heart Valve Dis. 1998;7:24-9.

5. Chauvaud S, Jebara V, Chachques JC, el Asmar B, Milaileanu S, Perier P, et al. Valve extension with glutaraldehyde-preserved autologous pericardium. Results in mitral valve repair. J Thorac Cardiovasc Surg. 1991;102:171-7.

6. Neethling WML, Yadav S, Hodge AJ, Glancy R. Enhanced biostability and biocompatibility of decellularized bovine pericardium, crosslinked with an ultra-low concentration monomeric aldehyde and treated with ADAPT. J Heart Valve Dis. 2008; 17:456-63; discussion 464.

7. Neethling WML, Glancy R, Hodge AJ. Mitigation of calcification and cytotoxicity of a glutaraldehyde-preserved bovine pericardial matrix: improved biocompatibility after extended implantation in the subcutaneous rat model. J Heart Valve Dis. 2010;19:778-85

8. Neethling W, Brizard C, Firth L, Glancy R. Biostability, durability and calcification of cryopreserved human pericardium after rapid glutaraldehydestabilization versus multistep ADAPT ${ }^{\circledR}$ treatment in a subcutaneous rat model. Eur J Cardiothorac Surg. 2014;45:e110-7.

9. James NL, van der Meer AL, Edwards GA, Edwards GA, Snelling SR, Begg JD, et al. Implantation of the VentrAssist Implantable Rotary Blood Pump in sheep. ASAIO J. 2003;49:454-8.

10. Junqueira LC, Bignolas G, Brentani RR. Picrosirius staining plus polarization microscopy, a specific method for collagen detection in tissue sections Histochem J. 1979;11:447-55.

11. Vorp DA, Schiro BJ, Ehrlich MP, Juvonen TS, Ergin MA, Griffith BP. Effect of aneurysm on the tensile strength and biomechanical behavior of the ascending thoracic aorta. Ann Thorac Surg. 2003;75:1210-4.

12. Stradins P, Lacis R, Ozolanta I, Purina B, Ose V, Feldmane L, et al. Comparison of biomechanical and structural properties between human aortic and pulmonary valve. Eur J Cardiothorac Surg. 2004;26:634-9.

13. Human P, Zilla P. Inflammatory and immune processes: the neglected villain of bioprosthetic degeneration? J Long Term Eff Med Implants. 2001;11:199-220.

14. Duran CM, Gometza B, Shahid M, Halees Al Z. Treated bovine and autologous pericardium for aortic valve reconstruction. Ann Thorac Surg. 1998;66(6 Suppl): S166-9.

15. Fricke TA, d'Udekem Y, Richardson M, Thyus C, Dronavalli M, Ramsay JM, et al. Outcomes of the arterial switch operation for transposition of the great arteries: 25 years of experience. Ann Thorac Surg. 2012;94:139-45.

16. Kumar SP, Prabhakar G, Kumar M, Kumar N, Shahid M, Ali ML, et al. Comparison of fresh and glutaraldehyde-treated autologous stented pericardium as pulmonary valve replacement. J Card Surg. 1995;10:545-51.

17. Myers PO, Tissot C, Christenson JT, Cikirikcioglu M, Aggoun Y, Kalangos A. Aortic valve repair by cusp extension for rheumatic aortic insufficiency in children: long-term results and impact of extension material. J Thorac Cardiovasc Surg. 2010;140:836-44. 